\title{
Radiative Heat Transfer on Nanofluids Flow Over a Porous Convective Surface in the Presence of Magnetic Field
}

\section{Kandasamy $\mathbf{R}^{*}$ and Mohamad $\mathbf{R}$}

Research Centre for Computational Mathematics, Faculty of Science, Technology and Human Development, Universiti Tun Hussein Onn Malaysia, Johor, Malaysia

\begin{abstract}
Nanofluids have been considered for applications as advanced heat transfer fluids for almost two decades. Nanofluid is a new kind of heat transfer medium, containing nanoparticles which are uniformly and stably distributed in a base fluid.Convection in nanofluids plays a key role in enhancing the rate of heat transfer either for heating or cooling nanodevices. In this paper, we investigate theoretically the impact of a convective surface on the heat transfer characteristics of water-based nanofluids over a static or moving wedge in the presence of magnetic field with variable stream condition. The governing nonlinear partial differential equations are made dimensionless with the similarity transformations. Numerical simulations are carried out through the very robust computer algebra software MAPLE 18 to investigate the effects of various pertinent parameters on the flow field. The results show that the temperature distribution in a nanofluid in the presence of thermal radiation with magnetic field significantly depends on the surface convection parameter. This finding is new and has not been reported in any open literature.
\end{abstract}

Keywords: Radiative heat transfer; Convective surface; Magnetic field; Nanofluids; Porous static or moving wedge

\section{Introduction}

The novel and advanced concepts of nanofluids offer fascinating heat transfer characteristics compared to conventional heat transfer fluids. There are considerable researches on the superior heat transfer properties of nanofluids especially on thermal conductivity and convective heat transfer. Applications of nanofluids in industries such as heat exchanging devices appear promising with these characteristics. The increases in effective thermal conductivity are important in improving the heat transfer behavior of fluids. Nanofluids, defined as fluids in which nanosized particles are suspended in a base liquid, are a novel types of working fluids used for heat transfer and cooling. Many of the publications on nanofluids about understand their behavior so that they can be utilized where straight heat transfer enhancement is paramount as in many industrial applications, nuclear reactors, transportation, electronics as well as biomedicine and food. Choi [1] first proposed the concept of using nanofluids to enhance thermal conductivity, these working fluids have shown great promise in heat transfer applications. Metal, metal oxides, ceramics and nonmetals such as carbon nanotubes and graphene can be used as nanoparticles in nanofluids, whereas water, ethylene glycol, oil and polymer solutions are generally used as base fluids. Several studies on nanofluids have been conducted by a number of researchers with respect to thermal conductivity enhancement and viscosity [2-5], phase change heat transfer [6-9] and convective heat transfer [1013].Ghasemi and Aminossadati [14], Congedo et al. [15] and Ho et al. [16] and [17] presented some numerical studies on the modeling of natural convection heat transfer in nanofluids using finite volume techniques or traditional finite difference methods.Magnetic nanofluid is a unique material that has both the liquid and magnetic properties. Many of the physical properties of these fluids can be tuned by varying magnetic field. In addition, they have been wonderful model system for fundamental studies.

Researchers have shown that nanofluids have not only better heat conductivity but also greater convective heat transfer capability than that of base fluids. Based on the radiative motion properties of nanoparticle, the utilization of nanofluids in thermal system becomes the new study hotspot. Radiative transport in porous media has important engineering applications in thermal energy collectors and the porous medium acts as a means to absorb or emit radiant energy that is transferred to or from a fluid. Generally, the fluid itself can be assumed to be transparent to radiation, because the dimensions for radiative heat transfer among the solid structure elements of the porous medium are usually much less than the radiative mean free path for scattering or absorption in the fluid. In this new age of energy awareness, our lack of abundant sources of clean energy and the widespread dissemination of battery operated devices, such as cell-phones and laptops, have accented the necessity for a smart technological handling of energetic resources.

Due to many engineering applications in aerodynamics, geothermal systems, crude oil extractions, ground water pollution, thermal insulation, heat exchanger, storage of nuclear waste, etc., convective flows over wedge shaped bodies have been extensively studied since the early formulation of the problem in 1931 by Falkner and Skan. They first studied two-dimensional flow of viscous incompressible fluid over a wedge. Since then many investigators have studied and reported results on wedge flow considering various flow conditions. Lin and Lin [18], Yih [19], Pantokratoras [20], Martin and Boyd [21], Rahman and Eltayeb [22], Rahman and Al-Hadhrami [23] and Muhaimin et al. [24]. Three properties that make nanofluids promising coolants are: (i) increased thermal conductivity, (ii) increased single-phase heat transfer, and (iii) increased critical heat flux. Research has shown that relatively small amounts of nanoparticles ( $5 \%$ or less volume fraction), can enhance thermal conductivity of the base fluid to a large extent. Therefore, exploiting the unique characteristics of nanoparticles, nanofluids are created with two features very important for heat

*Corresponding author: Kandasamy R, Research Centre for Computational Mathematics, Faculty of Science, Technology and Human Development, Universiti Tun Hussein Onn Malaysia, Johor, Malaysia, Tel:07-4537961; E-mail: ramasamy@uthm.edu.my

Received May 18, 2015; Accepted June 26, 2015; Published July 01, 2015

Citation: Kandasamy R, Mohamad R (2015) Radiative Heat Transfer on Nanofluids Flow Over a Porous Convective Surface in the Presence of Magnetic Field. J Appl Mech Eng 4: 172. doi:10.4172/2168-9873.1000172

Copyright: (C) 2015 Kandasamy R, et al. This is an open-access article distributed under the terms of the Creative Commons Attribution License, which permits unrestricted use, distribution, and reproduction in any medium, provided the original author and source are credited. 
transfer systems: (i) extreme stability, and (ii) ultra-high thermal conductivity. The effect of thermal radiation becomes important when a device operates in a high-temperature field. Radiative flows have important industrial, technological and geothermal applications such as high-temperature plasmas, cooling of nuclear reactors, liquid metal fluids, MHD accelerators, and power generation systems. A wide variety of industrial processes involve the transfer of heat energy. Throughout any industrial facility, heat must be added, removed, or moved from one process stream to another and it has become a major task for industrial necessity. These processes provide a source for energy recovery and process fluid heating/cooling.

In view of the above discussion the objective of the present investigation is to analyze the impact of a convective surface on the flow dynamics and radiative heat transfer characteristics of nanofluids over a static or moving wedge in the presence magnetic field. For a moving wedge its surface may be stretched or may be contracted. Thermal radiation, permeability of the porous medium, convective surface parameter, solid volume fraction of the nanofluid, magnetic parameter and suction/injection parameter on velocity and temperature fields for nanofluids using the thermophysical properties nanoparticles of the base fluid (water).

\section{Mathematical Analysis}

Consider the steady laminar two- dimensional flow of an incompressible viscous nanofluid of density $\rho_{f_{n}}$ past a moving or static porous wedge sheet with uniform velocity $U_{0}(x)=a x^{m}$ driven by the pressure gradient of the corresponding inviscid flow solution (Figure 1). We also consider influence of a constant magnetic field of strength $B_{0}$ which is applied normally to the sheet. The temperature at the lower surface of the wedge is heated by convection from a hot fluid (different from nanofluid)at a temperature $T_{0}$ which provides the heat transfer coefficient $h$ while the temperature of the moving or static porous wedge sheet $T_{\infty}$. It is further assumed that the induced magnetic field is negligible in comparison to the applied magnetic field (as the magnetic Reynolds number is small). The porous medium is assumed to be transparent and in thermal equilibrium with the fluid. Both the fluid and the porous medium are opaque for self-emitted thermal radiation. Also, the solar radiation is a collimated beam that is normal to the plate. The fluid is a water based nanofluid containing different types of nanoparticles: $\mathrm{Cu}, \mathrm{Al}_{2} \mathrm{O}_{3}, \mathrm{Ag}$ and $\mathrm{TiO}_{2}$. As mentioned before, the working fluid is assumed to have heat absorption properties. It is assumed that the base fluid and the nanoparticles are in thermal equilibrium and no slip occurs between them. $\frac{d p}{d x}=-a^{2} m \rho_{n f} x^{2 m-1}$. The coefficient $a$ is a function of the flow geometry. The exponent $m$ is a function of the wedge angle parameter $\beta=\frac{\Omega}{\pi}$ such as $m=\frac{\beta}{2-\beta}$. It is worth mentioning that the case $\beta=0$ corresponds to a horizontal plate, while $\beta=1$ corresponds to a vertical plate. In the present work, we consider only $0 \leq \beta \leq 1$. Under the above assumptions, the boundary layer equations governing the flow and thermal field can be written in dimensional form as

$$
\begin{aligned}
& \frac{\partial u}{\partial x}+\frac{\partial v}{\partial y}=0 \\
& \bar{u} \frac{\partial \bar{u}}{\partial \bar{x}}+\bar{v} \frac{\partial \bar{u}}{\partial \bar{y}}=\frac{1}{\rho_{f_{n}}}\left[U \frac{d U}{d x} \rho_{f_{n}}+\mu_{f_{n}} \frac{\partial^{2} \bar{u}}{\partial \bar{y}^{2}}+(\rho \beta)_{f_{n}} \bar{g}\left(T-T_{\infty}\right) \cos \Omega-\left(\sigma B_{0}^{2}+\frac{v_{f}}{K} \rho_{f_{n}}\right)(\bar{u}-U)\right] \\
& \bar{u} \frac{\partial T}{\partial \bar{x}}+\bar{v} \frac{\partial T}{\partial \bar{y}}=\alpha_{f_{n}} \frac{\partial^{2} T}{\partial \bar{y}^{2}}-\frac{1}{\left(\rho c_{p}\right)_{f_{n}}} \frac{\partial q_{r a d}^{\prime \prime}}{\partial y}
\end{aligned}
$$

Using Rosseland approximation for radiation (Sparrow and Cess [25], Rapits [26] and Brewster [27]) we can write $q_{r a d}^{\prime \prime}=-\frac{4 \sigma_{1}}{3 k^{*}} \frac{\partial T^{4}}{\partial y}$ where $\sigma_{1}$ is the Stefan-Boltzman constant is, $k^{*}$ is the mean absorption coefficient.

$$
\begin{aligned}
& \text { The boundary conditions of these equations are } \\
& u=U_{w}(x)=b x^{m}, v=V_{0},-k_{f_{n}} \frac{\partial T}{\partial y}=h\left(T_{0}-T\right) \text { at } y=0 ; \\
& \bar{u} \rightarrow U_{0}(x)=a x^{m}, T \rightarrow T_{\infty} \text { as } \bar{y} \rightarrow \infty
\end{aligned}
$$

Here $a$ and $b$ are constant, $\mathrm{u}$ and $v$ are the velocity components in the $\mathrm{x}$ and $\mathrm{y}$ directions, $B_{0}$ is uniform magnetic field strength, $\sigma$ is the electric conductivity, $T$ is the local temperature of the nanofluid, $\bar{g}$ is the acceleration due to gravity, $V_{o}$ is the velocity of suction/injection, $K$ is the permeability of the porous medium, $\rho_{f_{n}}$ is the effective density of the nanofluid, $q_{\text {rad }}^{\prime \prime}$ is the applied absorption radiation heat transfer, $\mu_{f_{n}}$ is the effective dynamic viscosity of the nanofluid, $\alpha_{f_{n}}$ is the thermal diffusivity of the nanofluid which are defined as ( see Aminossadati and Ghasemi [28]),

$$
\begin{aligned}
& \rho_{f_{n}}=(1-\zeta) \rho_{f}+\zeta \rho_{s}, \mu_{f_{n}}=\frac{\mu_{f}}{(1-\zeta)^{2.5}},(\rho \beta)_{f_{n}}=(1-\zeta)(\rho \beta)_{f}+\zeta(\rho \beta)_{s}, \alpha_{f_{n}}=\frac{k_{f_{n}}}{\left(\rho c_{p}\right)_{f_{n}}}, \\
& \left(\rho c_{p}\right)_{f_{n}}=(1-\zeta)\left(\rho c_{p}\right)_{f}+\zeta\left(\rho c_{p}\right)_{s} ; \frac{k_{f_{n}}}{k_{f}}=\frac{k_{s}+2 k_{f}-2 \zeta\left(k_{f}-k_{s}\right)}{k_{s}+2 k_{f}+2 \zeta\left(k_{f}-k_{s}\right)}
\end{aligned}
$$

where $\zeta$ is the nanoparticle volume fraction, $\mu_{f}$ is the dynamic viscosity of the base fluid, $\rho_{f}$ and $\rho_{s}$ are the densities of the base fluid and nanoparticle, $k_{f_{n}}$ is the thermal conductivity of the nanofluid, $\left(\rho c_{p}\right)_{f_{n}}$ is the heat capacitance of the nanofluid and $k_{\rho} k_{s}$ are the thermal conductivities of the base fluid and nanoparticle.

Following the lines of Kafoussias and Nanousis [29], the changes of variables are

$$
\eta=\sqrt{\frac{(m+1) U_{e}}{2 v_{f} x}} y, \psi=\sqrt{\frac{2 v_{f} x U_{e}}{m+1}} \text { and } \theta=\frac{T-T_{\infty}}{T_{0}-T_{\infty}}
$$

Where $v_{f}$ is the kinematic viscosity of the base fluid.By introducing the stream function $\psi$, which defined as $u=\frac{\partial \psi}{\partial y}$ and $v=-\frac{\partial \psi}{\partial x}$

Equations (1)-(3) take the non-dimensional form

$$
\begin{array}{r}
f^{\prime \prime \prime}-\frac{2}{m+1}(1-\zeta)^{2.5}\left[\left(M+\frac{\delta}{(1-\zeta)^{2.5}}\right)\left(f^{\prime}-1\right)-\left\{\left(1-\zeta+\zeta \frac{\left(\rho c_{p}\right)_{s}}{\left(\rho c_{p}\right)_{f}}\right)\right\} \gamma \cos \frac{\Omega}{2} \theta\right] \\
-\left(1-\zeta+\zeta \frac{\rho_{s}}{\rho_{f}}\right)(1-\zeta)^{2.5}\left[\frac{2 m}{m+1}\left(f^{\prime 2}-1\right)-f f^{\prime \prime}\right]=0 \\
\theta^{\prime \prime}+\frac{4}{3} \frac{k_{f}}{k_{f_{n}}} N\left\{\left(C_{T}+\theta\right)^{3} \theta^{\prime}\right\}^{\prime}-\operatorname{Pr}\left\{1-\zeta+\zeta \frac{\left(\rho c_{p}\right)_{s}}{\left(\rho c_{p}\right)_{f}}\right\} \frac{k_{f}}{k_{f_{n}}}\left[\frac{2}{m+1} f^{\prime} \theta-f \theta^{\prime}\right]=0
\end{array}
$$

where $\operatorname{Pr}=\frac{v_{f}}{\alpha_{f_{n}}}$ is the Prandtl number, $\delta=\frac{v_{f}}{a K}$ is the porous media parameter, $\gamma=\frac{g(\rho \beta)_{f} \Delta T}{\rho_{f} a^{2} b^{4} L}$ is the buoyancy or natural convection parameter, $M=\frac{\sigma B_{0}{ }^{2}}{\rho_{f} a k^{2}}$ is the magnetic parameter, $N=\frac{4 \sigma_{1} \theta_{w}^{3}}{k_{f} k^{*}}$ is the conductive radiation parameter and $C_{T}=\frac{T_{\infty}}{T_{0}-T_{\infty}}$ is the temperature ratio where $C_{T}$ assumes very small values by its definition as $T_{0}-T_{\infty}$ is very large compared to $T_{\infty}$. In the present study, it is assigned the value 
0.1. It is worth mentioning that $\gamma>0$ aids the flow and $\gamma<0$ opposes the flow, while $\gamma=0$ i.e., $\left(T_{0}-T_{\infty}\right)$ represents the case of forced convection flow. On the other hand, if $\gamma$ is of a significantly greater order of magnitude than one, then the buoyancy forces will be predominant. Hence, combined convective flow exists when $\gamma=O(1)$

The boundary conditions take the following form

$$
f^{\prime}(0)=\lambda, f(0)=S, \theta(0)=1+\frac{1}{\sqrt{2-\frac{2 m}{m+1}} B_{s}\left\{1-\zeta+\zeta \frac{\left(\rho c_{p}\right)_{s}}{\left(\rho c_{p}\right)_{f}}\right\}} \theta^{\prime}(0) \text { and } f^{\prime}=1, \theta \rightarrow 0 \text { as } \eta \rightarrow \infty
$$

where suction (or injection) parameter $S=\frac{-V_{0}}{\sqrt{\frac{m+1}{2}\left(\frac{U_{0} v_{f}}{x}\right)}}$ and relative velocity parameter $\lambda=\frac{U_{w}}{U_{0}}=\frac{b}{a}$ and the surface convection parameter $B_{S}=\frac{h x}{k_{f}} \operatorname{Re}_{x}^{-\frac{1}{2}}$.

For practical purposes, the functions $f(\eta)$ and $\theta(\eta)$ allow us to determine the skin friction coefficient and the Nusselt number as follows

$$
\begin{aligned}
& C_{f}=\frac{\mu_{f n}}{\rho_{f} U^{2}}\left(\frac{\partial u}{\partial y}\right)_{a t y=0}=-\frac{1}{(1-\zeta)^{2.5}}(\operatorname{Re} x)^{-\frac{1}{2}} f^{\prime \prime}(0) \\
& N u_{x}=\frac{x k_{f_{n}}}{k_{f}\left(T_{w}-T_{\infty}\right)}\left(\frac{\partial T}{\partial y}\right)_{a t y=0}=-(\operatorname{Re} x)^{\frac{1}{2}} \frac{k_{f_{n}}}{k_{f}} \theta^{\prime}(0)\left[1+\frac{4}{3} N\left(C_{T}+\theta(0)\right)^{3}\right]
\end{aligned}
$$

respectively. Here, $\operatorname{Re}_{x}=\frac{U_{w} x}{v_{f}}$ is the local Reynolds number.

The relative velocity parameter signifies the ratio of the wedge velocity to the velocity of the external free stream. The value $\lambda \neq 0$ corresponds to a moving wedge, whereas $\lambda=0$ corresponds to a static wedge. It can further be mentioned that $\lambda>0$ implies a stretching wedge surface in which both the surface and the free stream are moving along the same direction, whereas $\lambda<0$ implies the opposite case. The surface convection parameter or Biot number $B_{S}$ is a ratio of the hot fluid (the fluid supplying heat to the wedge surface) side convection resistance to the cold fluid (nanofluid) side convection resistance on a surface. For fixed cold fluid properties and fixed free stream velocity, the surface convection parameter at $B_{S}$ any $x$ station is directly proportional to the heat transfer coefficient $h$ associated with the hot fluid. The thermal resistance on the hot fluid side is inversely proportional to $h$. Hartee pressure gradient parameter $\left(m=\frac{\beta}{2-\beta}\right)$ is a function of the wedge angle parameter $\beta$ where the total angle of the wedge is $\beta \pi$. The value $m>0$ corresponds to accelerating flows whereas $m<0$ corresponds to decelerating flows. In the present investigation, we considered only accelerated flows.

\section{Results and Discussions}

The set of Equations (7) and (8) is highly nonlinear and it cannot be solved analytically and numerical solutions subject to the boundary conditions (9) are obtained using the very robust computer algebra software Maple 18. This software uses a fourth-fifth order Runge-Kutta-Fehlberg method as default to solve boundary value problems numerically using the dsolve command. For the benefit of the readers the Maple worksheet is listed in Appendix A. The transformed system of coupled nonlinear ordinary differential equations (7) and (8) including boundary conditions (9) depend on the various parameters. The numerical results are represented in the form of the dimensionless velocity and temperature. Numerical computations are carried out for several sets of values of the governing parameters, namely, magnetic parameter $(M)$, thermal radiation parameter $(N)$, convective surface parameter $\left(B_{S}\right)$, porosity parameter $(\delta)$, relative velocity parameter $(\lambda)$, nanoparticle volume fraction parameter $(\zeta)$ and suction if $S>0$ and injection if $S<0$. In order to illustrate the salient features of the model, the numerical results are presented in the following figures with fixed parameters $N=0.5, M=0.5, N=1.0, \lambda=1.0, \delta=0.5, B i=1.0, \operatorname{Pr}=0.6 .2, S=1.0$. The thermophysical properties of the base fluid and solid nanoparticles are given in Table 1, Oztop and Abu-Nada [30].

In Table 2, the present results of $f^{\prime \prime}(0)$ is compared with those of Yacob et al. [31] and Rahman et al. [32]. The results show a very good agreement with their results since the errors are found to be very less. This may be due to the fact that we have used Runge-Kutta-Fehlberg method (Maple 18 software) which has fifth-order accuracy. Thus the present results are more accurate than their results.

It is also observed from the Figure 2 that the agreement with the theoretical solution of velocity and temperature profiles for different values of convective surface parameter $B_{S}$ is excellent compared with Figure $3 \mathrm{~b}$ of Rahman et al. [32]. Figures $3 \mathrm{a}$ and $\mathrm{b}$ depict the influence of the suction $S$ on velocity and temperature profiles with uniform magnetic field in the presence of nanofluid flow over a convective surface. It is shown that the velocity of the nanofluid increases and the temperature of the nanofluid decreases with increase of suction strength $(S=0.0,0.5,1.0,2.0,3.0)$. The physical explanation for such a behavior is interesting to note that the heated fluid is pushed towards the convective surface where the buoyancy forces can act to retard the fluid due to high influence of the viscosity of the nanofluid. In the case of suction, the convective surface $\left(B_{S}=0.5\right)$ plays a physically significant role on the nanofluid flow and also the nanofluid is brought closer to the surface and enhances the hydrodynamic and reduces the thermal boundary layer thickness. This is due to the fact that the removal of the decelerated nanoparticles through the porous convective surface reduces for the growth of the thermal boundary layer. Decrease in thickness of the thermal boundary layer was caused in two ways: (i) the direct action of suction and (ii) the indirect action of suction of the nanofluid causing a thinner thermal boundary layer which corresponded to a higher temperature gradient, a consequent increase in the buoyancy force and a higher temperature gradient whereas the exact opposite behavior is observed by imposition of wall fluid injection.

\begin{tabular}{|l|c|c|c|c|}
\hline Physical properties & $\begin{array}{c}\text { Fluid phase } \\
\text { (water) }\end{array}$ & $\mathbf{C u}$ & $\mathbf{A l}_{2} \mathbf{O}_{\mathbf{3}}$ & $\mathrm{TiO}_{2}$ \\
\hline $\mathrm{C}_{\mathrm{p}}(\mathrm{J} / \mathrm{kg} \mathrm{K}$ & 4179 & 385 & 765 & 686.2 \\
\hline$\rho\left(\mathrm{kg} / \mathrm{m}^{3}\right)$ & 997.1 & 8933 & 3970 & 4250 \\
\hline $\mathrm{K}(\mathrm{W} / \mathrm{m} \mathrm{K})$ & 0.613 & 400 & 40 & 8.9538 \\
\hline
\end{tabular}

Table 1: Thermophysical properties of fluid and nanoparticles (Oztop and Abunada [30]).

\begin{tabular}{|c|c|c|c|c|}
\hline $\mathbf{m}$ & $\xi$ & Yacob et al. [31] & Rahman [32] & Present results \\
\hline 0.0 & 0.1 & 0.7179 & 0.7181 & 0.718094351 \\
\hline & 0.2 & 0.9992 & 0.9993 & 0.999265398 \\
\hline 0.5 & 0.1 & 1.5881 & 1.5882 & 1.588163987 \\
\hline & 0.2 & 2.2105 & 2.2106 & 2.105852436 \\
\hline 1.0 & 0.1 & 1.8843 & 1.8843 & 1.884257298 \\
\hline & 0.2 & 2.6226 & 2.6227 & 2.622647295 \\
\hline
\end{tabular}

Table 2: Comparison of the present results for $f^{\prime \prime}(0)$ with published works. 
Citation: Kandasamy R, Mohamad R (2015) Radiative Heat Transfer on Nanofluids Flow Over a Porous Convective Surface in the Presence of Magnetic Field. J Appl Mech Eng 4: 172. doi:10.4172/2168-9873.1000172

Page 4 of 7

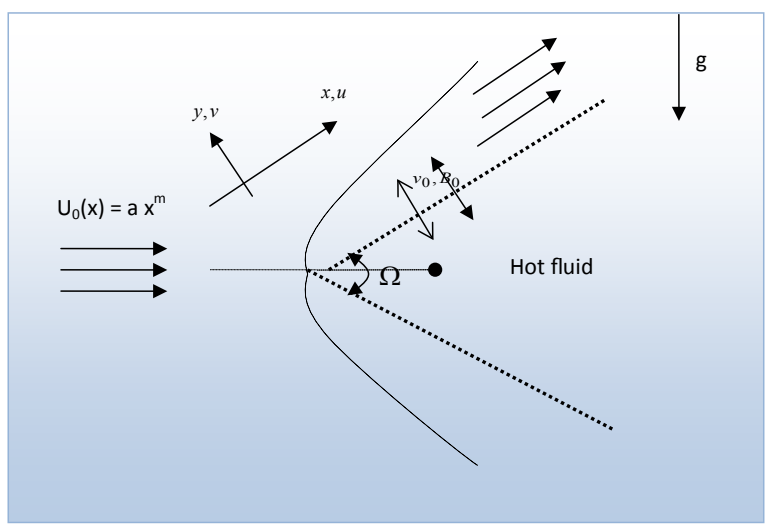

Figure 1: Physical flow model and Coordinate system over a porous wedge sheet.
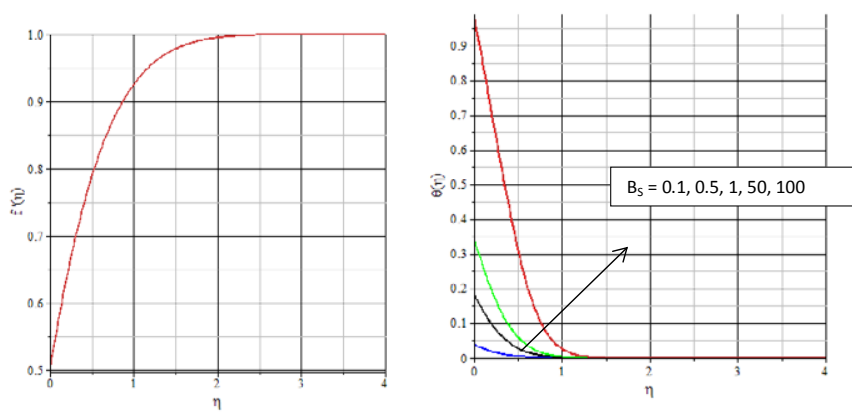

Figure 2: Coparison of convective surface effects on velocity and temperature profiles with Rahman et al. [32].

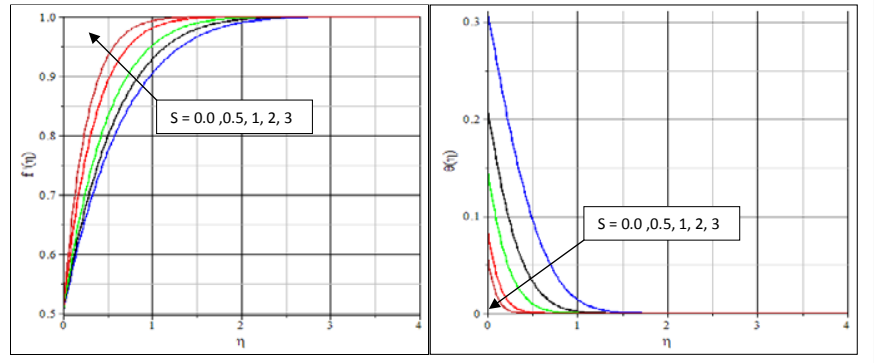

Figure 3: Suction effects on velocity and temperature profiles.

Illustrative velocity and temperature profiles of the nanofluid for five typical angles of inclination $\left(\Omega=0.0^{\circ}, 10.5^{\circ}, 23.5^{\circ}, 120^{\circ}\right.$ and $\left.180^{\circ}\right)$ are presented in Figures $4 \mathrm{a}$ and $4 \mathrm{~b}$. It is revealed that the temperature of the nanofluid is reduced by increasing the angle of inclination of convective wedge surface approximately corresponds to Hartee pressure gradient parameter $(S=0.0,0.5,1.0,2.0,3.0)$ because the angle of inclination increases the effect of the buoyancy force due to thermal diffusion decreases by a factor of $\cos \Omega$. Consequently the driving force to the fluid increases as a result temperature profiles decrease. From the Figures $4 \mathrm{a}$ and $4 \mathrm{~b}$, it is observed that the hydrodynamic boundary layer thickness of the nanofluid increases whereas thermal boundary layer thickness decreases as the angle of inclination increases. Similar to the effects of magnetic field, increasing the inclination angle makes it harder for the fluid to flow along the wedge plate and causes it to become warmer. This is due to the reduction in the thermal buoyancy effect caused by decrease in temperature and increase in velocity of the nanofluid. Due to the wedge plate is inclined from horizontal to vertical, the buoyancy effect on the velocity increasing and temperature of the nanofluid profiles decreasing. This is because the incident thermal radiation is initially absorbed by the absorbing fluid-matrix system which, in turn, heats up the ideally transparent plate. This operation of passing the absorbing fluid through an absorbing porous medium is believed to enhance thermal radiation collection by direct absorption in which heat losses are reduced as a result of plate temperature of the nanofluid. All these physical behavior are due to the combined effects of the strength of inclination of the wedge plate in the presence nanofluid with magnetic field.

In the presence of nanofluid flow along the convective wedge surface, the velocity profiles for different values of magnetic and porosity strength are shown in Figures 5 and 6 . It is observed from the Figure 5 that the velocity of the nanofluid increases with increase of the magnetic strength because of Lorentz force comes into action. This lorentz force acts in the opposite direction to the flow of nanofluid, opposing the motion of the nanofluid and tends to accelerates with increase of $M$. This is due to the combined effect of the increase of strength of the electrical conductivity and decrease of the density of the nanofluid in the presence of convective wedge surface. Due to the combined effect of decreasing of the permeability of the porous medium and increasing of the kinematic viscosity of the nanofluid, it is predicted from the Figure 6 that the velocity of the nanofluid increases with increase of porosity parameter.

Figure 7 presents the characteristic velocity and temperature
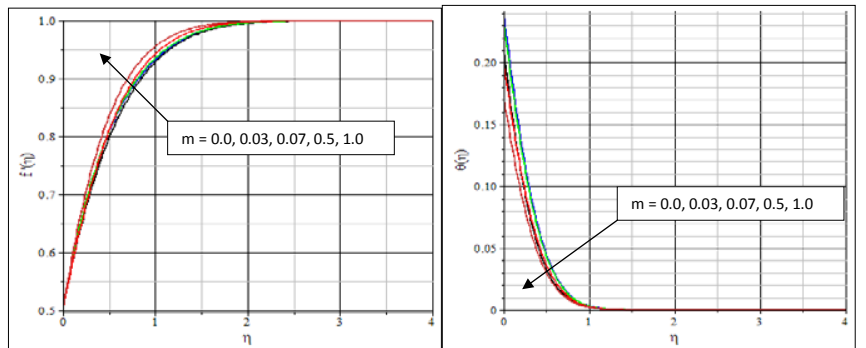

Figure 4: Hartee pressure gradient effects on velocity and temperature profiles.

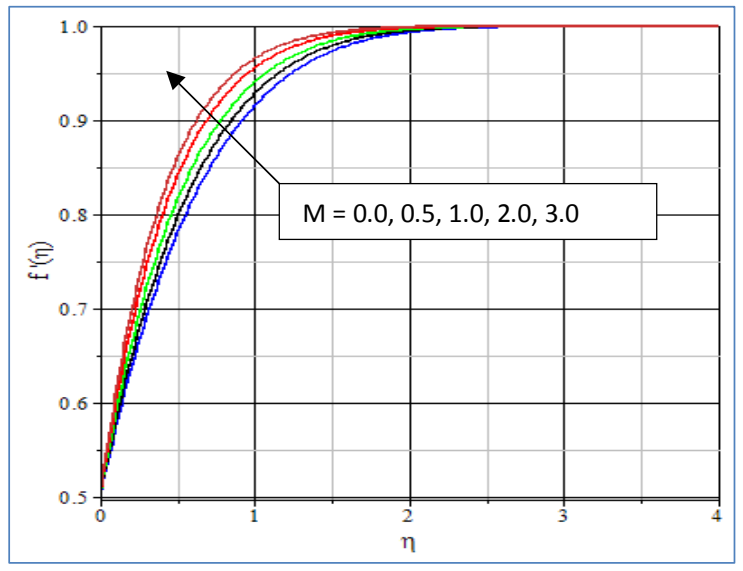

Figure 5: Magnetic effects on velocity profiles. 


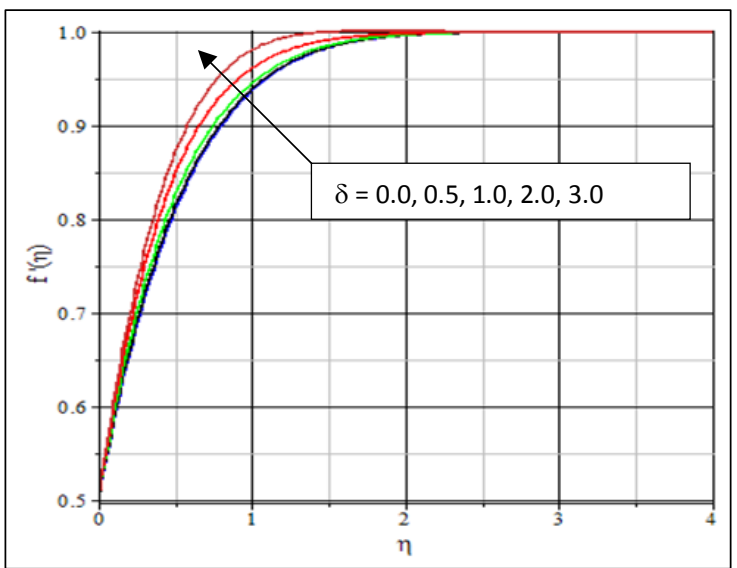

Figure 6: Porosity effects on velocity profiles.
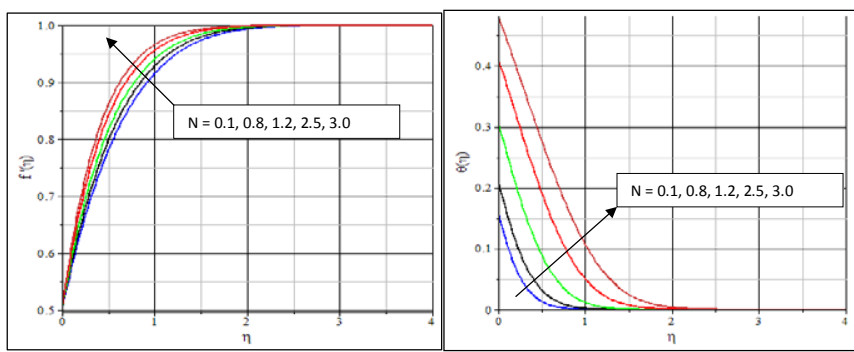

Figure 7: Thermal radiation effects on velocity and temperature profiles.

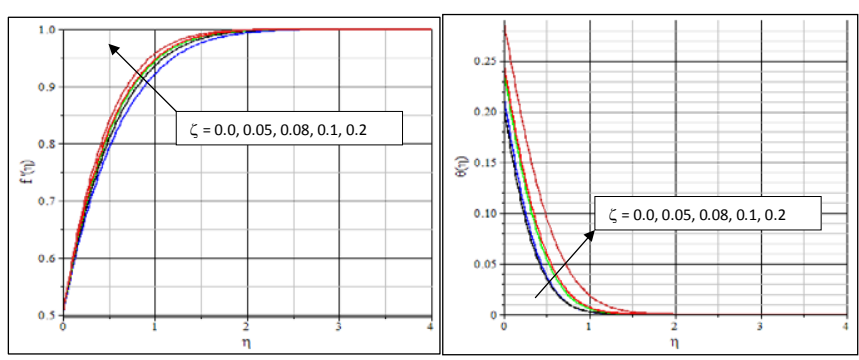

Figure 8: Nanoparticle volume fraction on velocity and temperature profiles.

profiles in the presence of nanofluid flow along the surface for different values of the thermal radiation parameter. According to Equations (2) and (3), the divergence of the radiative heat flux increases as thermal conductivity of the nanofluid $\left(k_{f}\right)$ raises which in turn increases the rate of radiative heat transfer to the nanofluid and hence the nanofluid velocity and temperature of the nanofluid accelerate. $N$ is to enhance the velocity and temperature of the nanofluid significantly in the flow region because of the combined effect of absorption and porosity of the convective surface in the presence magnetic field. This result can be explained by the fact that an increase in the values of $N=\frac{4 \sigma_{1} \theta_{w}^{3}}{k_{f} k^{*}}$ forgiven $k^{*}$ and $T_{\infty}$ means a decrease in the Rosseland radiation absorptive $k^{*}$. In view of this explanation, the effect of convective thermal radiation becomes more significant as $N \rightarrow 0(N \neq 0)$ and can be neglected when $N \rightarrow \infty$. It is noticed that the velocity and temperature of the nanofluid increases with increase of radiation parameter $N$. The increase in radiation parameter means the release of heat energy from the flow region and so the fluid temperature increases as the thermal boundary layer thickness becomes thicker. All these physical behavior are due to the combined effect of porous strength of the convective wedge surface and size of the nanoparticles.

Figure 8 displays the effects of volume fraction of nanoparticles on velocity and temperature distribution. In the presence of uniform thermal conductivity of the nanofluid along the convective porous wedge surface, it is note that the velocity and the temperature of the nanofluid increases with increase of the nanoparticles volume fraction parameter $\zeta$. It is also observed that the hydrodynamic and thermal boundary layer increase with increase of nanoparticle volume fraction parameter. This agrees with the physical behavior that when the volume fraction of nanoparticles increases the thermal conductivity and then the thermal and hydrodynamic boundary layer thickness increases. Variability of the size, shape, material, and volume fraction ofthe nanoparticles allows for tuning to maximize spectral absorption of thermal radiation throughout the fluid volume. Enhancement in the thermal conductivity can lead to efficiency improvements, although small, via more effective fluid heat transfer. Vast enhancements in convective surface area due to the extremely small particle size, which makes nano fluid-based thermal radiation systems attractive for thermochemical and photocatalytic processes.

Figure 9 illustrates typical profiles for velocity and temperature for different strength of convective surface in the presence of uniform magnetic field. In the presence of uniform porosity of the convective surface of the wedge, it is clearly shown that the velocity and temperature of the nanofluid increase with increase of the strength of convective surface parameter because of the combined effects of increase of heat transfer coefficient associated with the hot nanofluid and decrease of thermal conductivity of the nanofluid. In particular, the velocity and the temperature of the nanofluid gradually changes from lower value to the higher value only when the strength of convective surface parameter increases in the presence of nanofluid flow along the porosity of the wedge convective surface. For heat transfer characteristics mechanism about the connective surface, important result is the large distortion of the temperature field caused for $1<B_{S} \leq 50$. It is interesting to note that the characteristic of strength of convective surface plays a dominant role on heat transfer of the nanofluid. All these physical behaviors are due to the combined effects of the ratio of the hot fluid side convection resistance to the cold fluid (nanofluid) side convection resistance on a surface.

\section{Conclusions}

In the present paper, we have examined the performance of radiative heat transfer on water based nanofluid flow over a porous
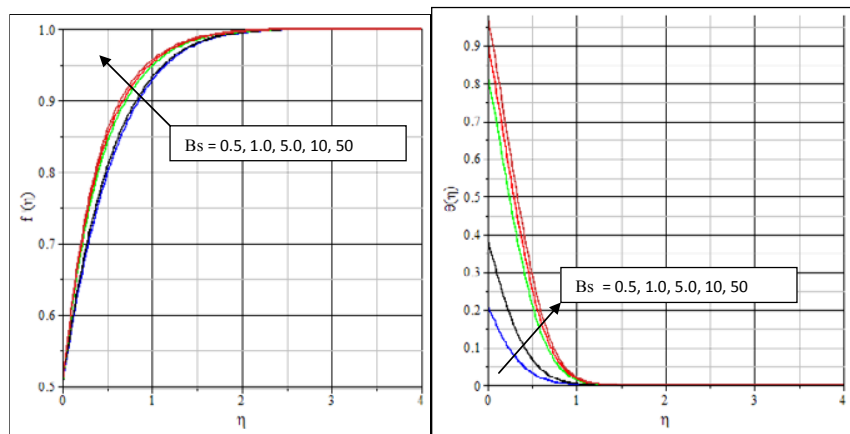

Figure 9: Thermal radiation effects on velocity and temperature profiles. 
static or moving convective wedge surface in the presence magnetic field with variable stream condition. The results of this study lead to the following conclusions:

- In the presence of uniform porosity of the convective surface of the wedge, it is clearly shown that the velocity and temperature of the nanofluid increase with increase of the strength of convective surface parameter because of the combined effects of increase of heat transfer coefficient associated with the hot fluid and decrease of thermal conductivity of the nanofluid. It is interesting to note that the characteristic of strength of convective surface plays a dominant role on heat transfer of the nanofluid.

- In the presence of uniform magnetic field, the velocity enhances and the temperature of the nanofluid reduces with increase of angle of inclination of the convective wedge surface approximately corresponds to Hartee pressure gradient. The fact is that as the angle of inclination increases the effect of the buoyancy force due to thermal diffusion increases by a factor of $\cos \Omega$ and consequently the driving force to the fluid increases as a result temperature decreases in the presence of water based nanofluid.

- Due to convective wedge surface, it is important to note that the velocity of the nanofluid increases whereas the temperature of the nanofluid decreases with increase of suction strength because the heated fluid is pushed towards the convective surface where the buoyancy forces can act to retard the fluid due to high influence of the viscosity of the nanofluid.

- Increase of thermal field due to increase in nanoparticle volume fraction parameter shows that the temperature increases gradually as we replace Copper by Alumina, and Titanium in the said sequence.

- It has been shown that mixing nanoparticles in a liquid (water based nanofluid) has a dramatic effect on the liquid thermophysical properties such as thermal conductivity. The porous convective wedge surface plays a very significant role on thermal boundary layer regime.

- It is interesting to predict that the velocity of the nanofluid increases with increase of the magnetic and porosity strength of the convective wedge surface because of the combined effect of increase of the strength of electrical conductivity and decrease of permeability of the porous medium and density of the nanofluid.

- It is noticed that the velocity and temperature of the nanofluid increase with increase of thermal radiation parameter. The increase in radiation parameter means the release of heat energy from the flow region and so the fluid motion and temperature of the nanofluid increases as the momentum and thermal boundary layer thickness enhance in the presence convective wedge surface.

- Nanofluids due to thermal radiation energy in the presence of magnetic field along the convective surface are important because they can be used in numerous applications involving heat transfer and other applications such as in detergency, solar collectors, drying processes, heat exchangers, geothermal and oil recovery, building construction, etc. The interdisciplinary nature of nanofluid research presents a great opportunity for exploration and discovery at the frontiers of nanotechnology. It is hoped that the present work will serve as a stimulus for needed experimental work on this problem.

\section{References}

1. Choi SUS (1995) Enhancing thermal conductivity of fluids with nanoparticles Developments and applications of non-newtonian flows, FED-231 66: pp. 99-105.
2. Kole M, Dey TK (2010) Viscosity of alumina nanoparticles dispersed in car engine coolant. Experimental Thermal and Fluid Science 34: 677-683.

3. Lee S, Choi SUS, Eastman JA (1999) Measuring thermal conductivity of fluids containing oxide nanoparticles, ASME Journal of Heat Transfer 121: 280-289.

4. Xuan Y, Li Q (2000) Heat transfer enhancement of nanofluids. International Journal of heat and fluid flow 21: 58-64.

5. Xie H, Wang J, Xi T, Liu Y, Ai F, et al. (2002)Thermal conductivity enhancement of suspensions containing nanosized alumina particles.Journal of Applied Physics 91: 4568-4572.

6. Das SK, Putra N, Roetze W (2003) Pool boiling characteristics of nano-fluids. International Journal of Heat and Mass Transfer 46: 851-862.

7. Das SK, Putra N, Roetzel W (2003) Pool boiling of nano-fluids on horizontal narrow tubes. International Journal of Multiphase Flow 29: 37-47.

8. You SM, Kim JH, Kim KH (2003) Effect of nanoparticles on critical heat flux of water in pool boiling heat transfer.Applied Physics Letters 83: 3374-3376.

9. Vassallo P, Kumar R, Amico SD (2004) Pool boiling heat transfer experiments in silica-water nano-fluids. International Journal of Heat and Mass Transfer 47 407-411.

10. Kang C, Okada M, Hattori A, Oyama K (2001) Natural convection of water-fine particle suspension in a rectangular vessel heated and cooled from opposing vertical walls (classification of natural convection in the case of suspension with a narrow-size distribution). International Journal of Heat and Mass Transfer 44: 73-82.

11. Putra N, Roetzel W, Das SK (2003) Natural convection of nano-fluids. International Journal of Heat and Mass Transfer 39: 775-784

12. Khanafer K, Vafai K, Lightstone M (2003) Buoyancy-driven heat transfer enhancement in a two-dimensional enclosure utilizing nanofluids. International Journal of Heat and Mass Transfer 46: 39-53.

13. Kim N, Kang YT, Choi CK (2004) Analysis of convective instability and heat transfer characteristics of nanofluids. Physics Fluids 16: 395-401.

14. Ghasemi B, Aminossadati SM (2009) Natural convection heat transfer in an inclined enclosure filled with a water-Cu nanofluid, Numer. Heat Transfer Part A Appl 55: 807-823.

15. Congedo PM, Collura S, Congedo PM (2009) Modeling and analysis of natura convection heat transfer in nanofluids.In: Proc. ASME Summer Heat Transfer Conf 3: 569-579.

16. Ho CJ, Chen MW, Li ZW (2008) Numerical simulation of natural convection of nanofluid in a square enclosure: Effects due to uncertainties of viscosity and thermal conductivity. Int J Heat Mass Transfer 51: 4506-4516.

17. Ho CJ, Chen MW, Li ZW (2007) Effect of natural convection heat transfer of nanofluid in an enclosure due to uncertainties of viscosity and therma conductivity.In: Proc. ASME/JSME Thermal Eng. Summer Heat Transfer Conf. HT 1: 833-841.

18. Lin HT, Lin LK (1987)Similarity solutions for laminar forced convection heat transfer from wedges to fluids of any Prandtl number. Int J Heat Mass Transfer 30: 1111-1118.

19. Yih KA (1998) Uniform suction/blowing effect on the forced convection about a wedge: Uniform heat flux. Acta Mech 128: 173-181.

20. Pantokratoras A (2006) The Falkner-Skan flow with constant wall temperature and variable viscosity. Int J Thermal Sci 45: 378-389.

21. Martin MJ, Boyd ID (2010) Falkner-Skan flow over a wedge with slip boundary conditions. J Thermophys Heat Transf 24: 263-270.

22. Rahman MM, Eltayeb IA (2011) Convective slip flow of rarefied fluid over a wedge with thermal jump and variable transport properties. Int J Thermal Sci 50: $468-479$

23. Rahman MM, Al-Hadhrami AMK (2012) Nonlinear slip flow with variable transport properties over a wedge with convective surface.Stavrinides SG (Ed.), Chaos and complex systems, 21 Springer, Berlin.

24. Muhaimin I, Kandasamy R, Khamis AB, Roslan R (2013) Effect of thermophoresis particle deposition and chemical reaction on unsteady MHD mixed convective flow over a porous wedge in the presence of temperaturedependent viscosity.Nucl Eng Des 261: 95-106. 
Citation: Kandasamy R, Mohamad R (2015) Radiative Heat Transfer on Nanofluids Flow Over a Porous Convective Surface in the Presence of Magnetic Field. J Appl Mech Eng 4: 172. doi:10.4172/2168-9873.1000172

Page 7 of 7

25. Sparrow EM, Cess RD (1978) Radiation heat transfer, Washington: Hemisphere.

26. Raptis A (1998) Radiation and free convection flow through a porous medium. Int Comm Heat Mass Transfer 25: 289-295.

27. Brewster MQ (1972) Thermal Radiative Transfer Properties, John Wiley and Sons.

28. Aminossadati SM, Ghasemi B (2009) Natural convection cooling of a localized heat source at the bottom of a nanofluid-filled enclosure. Eur J Mech B/Fluids 28: $630-640$.

29. Kafoussias NG, Nanousis ND (1997) Magnetohydrodynamic laminar boundary layer flow over a wedge with suction or injection. Canadian Journal of Physics $75: 733$

30. Oztrop, Abu-nada (2008) Numerical study of natural convection in partially heated rectangular enclosures filled with nanofluids. Int J Heat Fluid Flow 29 : 1326-1336.

31. Yacob NA, Ishak V, Pop I (2011) Falkner-Skan problem for a static or moving wedge in nanofluids. Int $\mathrm{J}$ Thermal Sci 50: 133-139.

32. Rahman MM, Al-Mazroui WA, Al-Hatmi FS, Al-Lawtia MA, Eltayeb IA (2014) The role of a convective surface in models of the radiative heat transfer in nanofluids. Nuclear Engineering and Design 275: 382-392. 